\title{
Primary effusion lymphoma (PEL) in a renal transplant patient
}

\author{
Authors: Raghda Noureldin, ${ }^{\mathrm{A}}$ Syed Athar, ${ }^{\mathrm{A}}$ Vidya Nagrale ${ }^{\mathrm{A}}$ and Waqar Gaba ${ }^{\mathrm{A}}$
}

\section{Introduction}

Primary effusion lymphoma (PEL) is a lymphoma of large B cells that primarily presents as effusions without a tumour mass. ${ }^{1,2}$ PEL occurs in immunodeficient states such as acquired immunodeficiency syndrome (AIDS) or with use of immunosuppressant medications, and is associated with human herpesvirus-8 (HHV-8) and Epstein-Barr virus $(\mathrm{EBV}){ }^{2,3}$ We report a case of primary effusion lymphoma 15 years after renal transplant, presenting as exudative ascites.

\section{Case presentation}

An 81-year-old African woman presented with a history of weight loss and abdominal distention of 2 weeks' duration. The patient underwent renal transplantation 15 years ago; the aetiology of her renal failure was hypertension on immunosuppressant agents including mycophenolate mofetil and cyclosporine. She also has a history of heart failure with preserved ejection fraction, persistent atrial fibrillation on apixaban, hypertension, dyslipidaemia and severe pulmonary arterial hypertension. The patient had been treated with right-sided thoracentesis for pleural effusion 2 years prior to presentation; cytology was suggestive of high-grade lymphoproliferative disease. She was lost to follow-up for further workup since then. Physical examination was positive for ascites, with no palpable masses, lymph nodes or other significant findings. Lab tests showed normocytic normochromic anaemia, mild neutropenia and hyperosmolar hyponatraemia; polymerase chain reaction test was negative for HIV, cytomegalovirus (CMV) and EBV. Computed tomography (CT) of her neck/chest/abdomen and pelvis were consistent with ascites in all abdominal quadrants; no solid masses were reported. Ascitic fluid was exudative on analysis, with peritoneal protein of $68 \mathrm{~g} / \mathrm{L}$ and lactate dehydrogenase of 2,559 IU/L. Bacterial and tuberculosis cultures of ascitic fluid were negative. On cytology, lymphoma cells were found to be positive for multiple immunohistochemical stains including CD38, CD30, epithelial membrane antigen (EMA) and HHV-8, but negative for CD138, CD20 and PAX-5. An EBVencoded small ribonucleic acid (EBER) chromogenic in situ

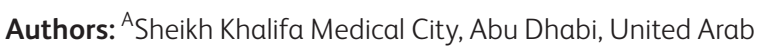
Emirates

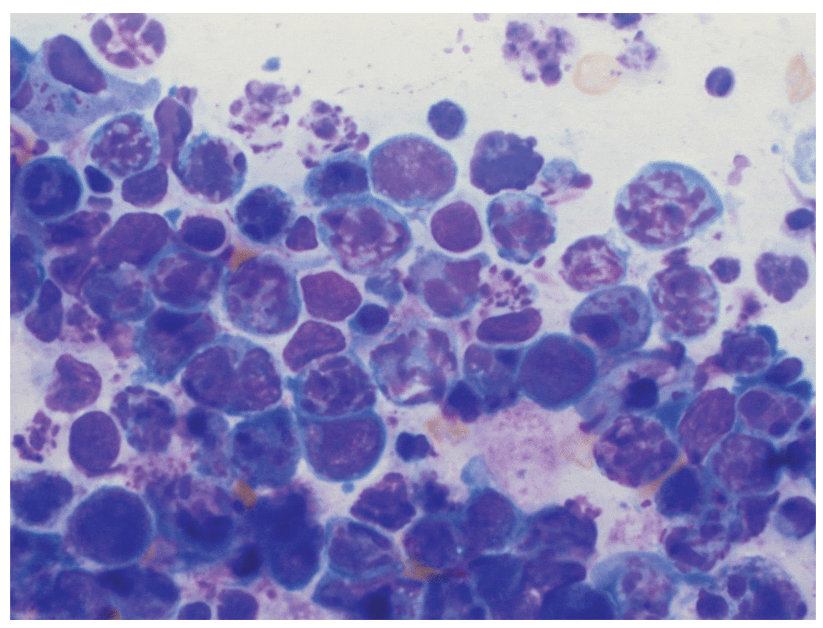

Fig 1. Cytospin of the paracentesis fluid (Giemsa stain; original magnification $\times 100$; oil immersion).

hybridisation study was negative. Concurrent flow cytometry showed an abnormal B-lymphoma population positive for CD45 and CD38, and negative for B, T and myeloid markers. Fig 1 shows a cytospin of the paracentesis fluid, showing a large, pleomorphic lymphoma cell with basophilic cytoplasm. Based on the above histopathological and flow cytometry findings, PEL was diagnosed. A plan of management was discussed in the presence of the patient's family, haematology and internal medicine teams; the family was aware of the nature of the disease, and the poor prognosis associated with it. Active versus palliative care was discussed with risks and benefit of treatment explained. The patient opted for palliative management as per family preference, with no further investigations or treatment to be pursued.

\section{Conclusion}

This case is a rare presentation of PEL presenting as exudative ascites in the setting of immunosuppression in an organ transplant recipient from the development of an $\mathrm{HHV}-8$ infection. This highlights the importance of considering PEL as a possible diagnosis in HIV-seronegative older patients receiving long-term immunosuppressant medications. 


\section{Conflicts of interest}

None declared.

\section{References}

1 Broaddus VC, Mason RJ, Ernst JD et al (eds). Murray and Nadel's Textbook of Respiratory Medicine, 6th edn. Philadelphia: Elsevier Saunders, 2016.
2 Sultana S, Al Salihi S, Tandon N et al. Post-transplant lymphoproliferative disorder presented in a form of primary effusion lymphoma with t $(8 ; 14)$. Ann Clin Lab Sci 2017;47:344-8.

3 Kugasia IAR, Kumar A, Khatri A et al. Primary effusion lymphoma of the pleural space: report of a rare complication of cardiac transplant with review of the literature. Transpl Infect Dis 2019;21:e13005. 